\title{
Opiate withdrawal: inpatient versus outpatient programmes and preferred versus random assignment to treatment
}

\author{
MICHAEL GOSSOP, ANDREW JOHNS, LYNETTE GREEN
}

\begin{abstract}
The relative effectiveness of an inpatient and an outpatient withdrawal programme for opiate addicts was studied. Forty five men and 15 women (mean age 26.13 (SD 5.12) years) took part in the study, all of whom were voluntary patients at the drug dependence clinic of the Maudsley Hospital in London between 1984 and 1985. Subjects were asked if they were prepared to accept either inpatient or outpatient withdrawal, and those who were willing to do so were assigned randomly to the randomised outpatient group or the randomised inpatient group. Those who expressed a strong preference were assigned, as appropriate, to the preferred outpatient group or the preferred inpatient group. Addicts in the inpatient group were more likely to achieve complete withdrawal ( 25 out of $31,81 \%$ ) than those in the outpatient group (five out of $29,17 \%$ ). This difference could not be attributed to pretreatment factors related to drugs or to social or psychological differences between the groups.
\end{abstract}

These results have clinical and policy implications for the treatment of opiate addicts.

\section{Introduction}

Detoxification (supervised withdrawal) is generally seen as a preliminary step in the treatment of opiate addiction. The most commonly used withdrawal procedure for opiate addicts entails gradually decreasing doses of oral methadone. This is agreed to be the most effective and safe technique currently available. ${ }^{12}$

It is surprising, therefore, that there is little agreement on how this procedure should be carried out. In particular, there is disagreement about whether withdrawal should be attempted on an inpatient or outpatient basis. This question is not merely of interest to medical scientists but has important implications related to health care policy as it is considerably cheaper and more cost effective to run an outpatient service. In the related problem of alcoholism Edwards and Guthrie found no difference in effectiveness between an inpatient and an outpatient programme. ${ }^{3}$ If this were also true of opiate dependence it would have important implications for the delivery of services.

In this study we considered two specific questions: whether opiate addicts are more likely to achieve abstinence on an inpatient or an outpatient basis, and whether the addict's preference for withdrawal in an inpatient or outpatient programme affected the outcome.

\section{Subjects}

Sixty subjects took part in this study ( 45 men and 15 women, mean age $26 \cdot 13$ (SD 5.12) years). All were voluntary patients at the drug dependence clinic of the Maudsley Hospital in London between 1984 and 1985. All were physically dependent on opiates and were asking to be withdrawn.

\footnotetext{
Drug Dependence Clinical Research and Treatment Unit, The Bethlem Royal Hospital, Beckenham, Kent BR3 3BX

MICHAEL GOSSOP, BA, PHD, principal psychologist

ANDREW JOHNS, BSC, MRCPSYCH, honorary senior registrar LYNETTE GREEN, BA, research psychologist

Correspondence to: Dr Gossop.
}

Most of the subjects (47 (78\%)) were primarily dependent on illicit heroin; the remainder were dependent on either methadone (11 subjects, $18 \%$ ) or other opioid drugs such as codeine or dihydrocodeine phosphate (DF118) (two subjects, 3\%). The main source of supply was the black market (48 subjects, $80 \%$ ). Of the 60 subjects, 31 were intravenous users, 17 smoked heroin, and 12 were oral users $(52 \%, 28 \%$, and $20 \%$, respectively). Twenty one subjects claimed to be using other drugs no more than twice weekly. Most subjects (39) used various other non-opiate drugs.

\section{Methods}

Subjects were assigned to one of four groups: the randomised outpatient group, the randomised inpatient group, the preferred outpatient group, and the preferred inpatient group. All subjects were asked if they were prepared to accept either inpatient or outpatient withdrawal. Those subjects who were willing to accept either (20) were then assigned randomly to one of the two randomised groups. The 40 subjects who expressed a strong preference for inpatient or outpatient withdrawal were assigned to the appropriate preference group. The smaller sample size of the randomised groups reflects the greater difficulty experienced in finding subjects who had no clear preference and who were willing to be assigned randomly.

The withdrawal phase of the inpatient treatment programme lasted for 21 days, although this is only the first stage of a longer treatment and rehabilitation programme. The outpatient withdrawal programme lasted for eight weeks and entailed weekly attendance at the clinic for counselling and support by a psychiatrist. Oral methadone was supplied by prescription to the addict on a daily basis through a chemist (pharmacy). The 21 days of the inpatient withdrawal and the eight weeks of the outpatient withdrawal were the durations routinely used by the clinic for such programmes: we considered it to be artificial to force both programmes into the same period merely to give the appearance of methodological neatness if this was not also consistent with clinical experience. Both withdrawal schemes used oral methadone, the dose of which was reduced on a daily basis using a linear (equal dose) reduction model.

The principal aim was to achieve abstinence at the end of the supervised withdrawal regimen. Urine specimens were collected before and throughout the trial, and abstinence was confirmed by urine analysis.

\section{Results}

A comparison of the inpatient and outpatient groups showed that inpatients were more likely to achieve complete withdrawal $\left(\chi^{2}=21 \cdot 6\right.$, $\mathrm{p}<0.001)$. Of the 31 patients in the inpatient withdrawal group, $25(81 \%)$ were successfully withdrawn from opiates (and all other drugs, including alcohol), and of the 29 outpatients, 5 (17\%) were successfully withdrawn from opiates.

We also compared the other main treatment factor: whether the patient was randomly assigned to treatment or had chosen a particular option. This failed to show any significant effect at the $5 \%$ level, although subjects who expressed a clear preference tended to do better than those who expressed no preference and were assigned to inpatient or outpatient withdrawal at random $\left(\chi^{2}=2 \cdot 7, p=0 \cdot 10\right)$. Twenty three of the 40 patients in the preferred group $(58 \%)$ were successfully withdrawn, compared with only seven of the 20 patients in the random group (35\%).

There were no differences between the two groups in terms of opiate dose before withdrawal (mean for total sample $0 \cdot 25-0.5 \mathrm{~g}$ of illicit heroin or $30-40 \mathrm{mg}$ of methadone), dose of methadone required for withdrawal (mean $37.5 \mathrm{mg}$ ), age at which opiates were first used (mean 20.7 years), age at which addiction began (mean 22.5 years), previous experience of supervised withdrawal programme (most subjects were attending their first programme), and daily use of alcohol (five inpatients, three outpatients). No differences were found in such measures of social functioning as forensic history, number of acquaintances who used drugs, and social supports to help with withdrawal.

The reasons for failure in those patients who did not maintain contact with the clinic are unknown. In those cases where this information is available withdrawal symptoms were mentioned more often by outpatients, as were 
pressures related to situation and the influence of other drug users. In the outpatient group the most risky times appeared to be at the beginning and end of the withdrawal schedule. Six subjects ( $21 \%$ of this group) failed in the first 14 days of the eight week programme, and $9(31 \%)$ failed in the last week. Of the six inpatients who failed, two did so on the first day and the four others in the last six days.

The outpatient sample was more likely to remain in contact with the hospital's drug dependence services $\left(\chi^{2}=9 \cdot 19, \mathrm{p}<0 \cdot 05\right)$; 16 out of $29(55 \%)$ received further counselling or treatment from the clinic after their attempt at withdrawal whereas 22 out of $37(71 \%)$ of the inpatients lost contact with the clinic. A further analysis of the data for all subjects showed that those addicts who had been successfully withdrawn from drugs were less likely to remain in immediate contact with the clinic and took longer to re-establish contact $(t=2 \cdot 38, \mathrm{p}<0.05)$.

\section{Discussion}

This study was concerned with comparing methods of withdrawing opiate addicts from their drugs and was not a study of treatments for addiction in the wider sense. "Success" was defined in terms of becoming completely free of drugs. As Newman has argued, prolonged abstinence is not an appropriate goal of detoxification; the basic goal is elimination of the acute neurophysiological dependence. $^{4}$

The main finding of this study was that a supervised inpatient withdrawal was more successful than the outpatient scheme. Compared with the 25 inpatients who succeeded (81\%), only five of the outpatients completed their withdrawal from opiates (17\%). This outcome could not be attributed to pretreatment factors related to drugs, as the two groups had virtually identical patterns of drug misuse. In addition, there were no appreciable differences between the groups in a wide range of social factors.

The superior success of inpatient withdrawal is not entirely surprising, as the inpatient programme entailed much more supervision of all aspects of the patients' behaviour as well as higher levels of support and therapy. Although Edwards and Guthrie showed that outpatient treatment for alcoholism was just as effective as an inpatient programme, their study compared inpatient treatment with an intensive form of outpatient care. ${ }^{3}$ The inpatient treatment options in our study and that of Edwards and Guthrie may be regarded as broadly similar, but in our study the outpatient withdrawal programme was non-intensive and no community support was provided outside the clinic.

As the inpatient and outpatient withdrawal periods were different ( 21 days for inpatients and 56 days for outpatients) it could be suggested that the difference in the results may have been due to this. The data do not, however, support this suggestion. Of the outpatients, 9 (31\%) failed within the first 21 days. Most subjects who completed the first 21 days of the outpatient programme continued to withdraw until the final week, when nine more dropped out or began using illicit drugs again. There was a similarly high failure rate at the beginning and end of both the inpatient and the outpatient programmes. Of the 30 subjects who failed in both programmes, $21(70 \%)$ did so in the first and last quarters of the withdrawal periods. It has been shown elsewhere that anxiety plays a large part in the addict's response to opiate withdrawal, ${ }^{5}$ and fear of withdrawal symptoms or fear about being free of drugs may have produced the result observed in this study.

The effect of patient preference for either inpatient or outpatient withdrawal was not significant. The complete failure of the randomised outpatient group suggests, however, that inpatient options should be preferred (if available) unless the addict has strong preferences for outpatient withdrawal.

These results show that opiate addicts can be withdrawn with a satisfactory level of success on an inpatient basis. It is not clear, however, what the wider implications are for outpatient withdrawal schemes. One study, for instance, suggested that a $24 \%$ abstinence rate for outpatient withdrawal might be regarded as acceptable. ${ }^{6}$ Also, the present results should not be overgeneralised as representative of all outpatient schemes. In this study the outpatient programme was non-intensive and clinic based. Other schemes that offer a more intensive package or include community support might well produce a higher success rate. In view of the drastic shortage of inpatient beds for opiate addicts in Britain and because the financial costs of outpatient withdrawal are much lower than those of inpatient withdrawal, the possibilities of improving outpatient options deserve further exploration.

We thank Dr P H Connell, director of the drug dependence clinical research and treatment unit, for allowing his facilities to be used for this study.

\section{References}

1 Kleber HD. Detoxification from narcotics. In: Lowinson JH, Ruiz P, eds. Substance abuse: clinical problems and perspectives. Baltimore: Williams and Wilkins, 1981

2 Lipton DS, Maranda MJ. Detoxification from heroin dependence: an overview of method and effectiveness. In: Stimmel B, ed. Evaluation of drug treatment programmes. New York: Haworth Press, 1983.

3 Edwards G, Guthrie S. A controlled trial of inpatient and outpatient treatment of alcohol dependence. Lancet 1967; ; 555 .

4 Newman RG. Detoxification treatment of narcotic addicts. In: Dupont RI, Goldstein A, O'Donnell JA, eds. Handbook on drug abuse. Washington, DC: US Government Printing Office, 1979.

5 Phillips GT, Gossop M, Bradley B. The influence of psychological factors on the opiate withdrawal syndrome. Br 7 Psychiatry (in press).

6 Renner JA, Rubin ML. Engaging heroin addicts in treatment. Am f Psychiatry 1973;130:976.

(Accepted 6 May 1986)

\section{SHORT REPORTS}

\section{Effect of dietary supplementation with fish oil on systolic blood pressure in mild essential hypertension}

MaxEPA is an oil derivative of marine fish rich in $\omega 3$ polyunsaturated fatty acids eicosapentaenoic acid (20:5 $\omega 3)$ and docosahexaenoic acid (22:6 133$)$, which significantly reduce systolic blood pressure in both normal volunteers ${ }^{12}$ and patients undergoing haemodialysis. ${ }^{3}$ No data exist on the effect of dietary supplementation with fish oil in patients with hypertension.

\section{Patients, methods, and results}

Eight men and eight women aged $45-74$ (mean 54.8 ) years in whom the supine diastolic pressure was $90-110 \mathrm{~mm} \mathrm{Hg}$ and systolic pressure below $200 \mathrm{~mm} \mathrm{Hg}$ after a two month run in period without treatment were randomly assigned to double blind treatment with MaxEPA $16.5 \mathrm{~g} /$ day or indistinguishable placebo capsules. Patients crossed over to the alternative treatment after six weeks. Resting blood pressure (diastolic pressure taken as Korotkoff phase V) was recorded by the same observer on each occasion. Blood was taken for measurement of fibrinogen concentration, factor VIII related antigen, platelet count, mean platelet volume, and platelet aggregation induced by $0.8 \mathrm{nmol}, 2 \mathrm{nmol}$, and $5 \mathrm{nmol}$ adenosine diphosphate and $0.19 \mathrm{mg}$ collagen $/ \mathrm{ml}$ after the initial run in period and at the end of each six week treatment period.

One 60 year old man was withdrawn while receiving placebo, before receiving MaxEPA, as his systolic blood pressure had risen above $200 \mathrm{~mm} \mathrm{Hg}$. He was excluded from the statistical analysis, which was performed using the paired Student's $t$ test.

The mean blood pressure before randomisation was $160 / 94 \mathrm{~mm} \mathrm{Hg}$. Mean blood pressure after six weeks' placebo treatment was $161 / 94.5 \mathrm{~mm} \mathrm{Hg}$ and after six weeks' treatment with MaxEPA $151 / 92.5 \mathrm{~mm} \mathrm{Hg}$. Lying systolic pressure was lower after treatment with MaxEPA than after treatment with placebo by a mean $5.84 \%(p<0.02)$ (figure), and standing systolic was a mean $5.66 \%$ lower after MaxEPA $(p<0.05)$. The lower mean diastolic pressure observed after treatment with MaxEPA did not reach significance. There was no significant difference in systolic or diastolic pressures after the wash out period and placebo treatment 